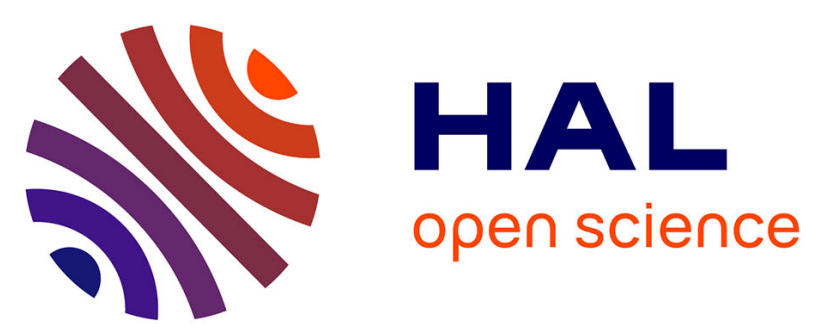

\title{
The use of chemical potential to describe water transfer in complex media with strong solid-liquid bonding
}

\author{
M. Anoua, Alejandra Ramirez-Martinez, Fabien Cherblanc, Jean-Claude
}

Benet

\section{- To cite this version:}

M. Anoua, Alejandra Ramirez-Martinez, Fabien Cherblanc, Jean-Claude Benet. The use of chemical potential to describe water transfer in complex media with strong solid-liquid bonding. Transport in Porous Media, 2014, 102, pp.111-122. 10.1007/s11242-013-0265-4 . hal-00979333

\section{HAL Id: hal-00979333 \\ https://hal.science/hal-00979333}

Submitted on 15 Apr 2014

HAL is a multi-disciplinary open access archive for the deposit and dissemination of scientific research documents, whether they are published or not. The documents may come from teaching and research institutions in France or abroad, or from public or private research centers.
L'archive ouverte pluridisciplinaire HAL, est destinée au dépôt et à la diffusion de documents scientifiques de niveau recherche, publiés ou non, émanant des établissements d'enseignement et de recherche français ou étrangers, des laboratoires publics ou privés. 
1 The use of chemical potential to describe water transfer in

2 complex media with strong solid-liquid bonding

3

\author{
Anoua $^{1}$, M., Ramirez-Martinez ${ }^{2}$, A., Cherblanc ${ }^{2}$, F., Bénet, J.C ${ }^{2}$. \\ ${ }^{1}$ Faculté des Sciences Département de Physique Route Ben Maachou, 24000, El \\ Jadida, Maroc. \\ ${ }^{2}$ Laboratoire de Mécanique et Génie Civil, UMR CNRS 5508, Université Montpellier II - cc \\ 048, Place E. Bataillon - 34095 Montpellier Cedex 5.
}

\begin{abstract}
We consider a complex medium composed of finely intertwined micro-skeletons and microcompartments where water transfer can occur. In these media, at low moisture content, water pressure measurement is not longer possible. Mass transfer is then expressed in terms of chemical potential gradient. The assumption of local thermodynamic equilibrium, resulting in the uniformity of water chemical potential in all microstructures, is essential to define a sorption isotherm reflecting the relationship between water activity and average moisture content. In this case, it is also possible to describe water transfer by using the chemical potential gradient. Radial water transfer in wood is examined using a destructive method for calculating water flux and chemical potential gradient at the same position and at the same time. We deduce the variation of transport coefficient as a function of moisture content.
\end{abstract}

\title{
Keywords
}

Local thermodynamic equilibrium, transport coefficient, isotherm, wood, water flux, water diffusion.

\section{Introduction}

Biological tissue, gels, food products and soils are characterized by the juxtaposition of micro-skeletons (continuous solid or granular phases, network of polymers, membranes, cell walls), and micro-compartments (interstitial fluid, films, gas bubbles, micro cracks and intracellular phases). These micro-structures are of various shapes and sizes. Subsequently, the scale of these microstructures will be called $\alpha$. For example, figure 1 shows the assembly of microstructures and micro-compartments in the case of two media: agar gel and coffee endosperm. For these products the scale $\alpha$ ranges from a micron to a few dozen microns. The macroscopic scale, noted $\beta$, corresponds to the assembly of a large number of $N$ microstructures, such that any fluctuation around $N$ does not change the properties measured at this scale. The existence of wide interfaces modifies the physical properties of certain constituents, in particular of water. This is due to the physical interactions between the constituents of liquid phase and solid phase. For a given porous media, the energy of interaction increases when the distance from the molecules of water to the surface decreases, and then when the water content decreases. 
Filtration of liquid water in porous media at $\beta$ is generally described by Darcy's law. This law was initially introduced to describe the flow in saturated soils and then extended to other situations by introducing matric, gravitational, and osmotic potentials [Corey \& Klute, 1985; Niato \& Bear, 1996]. Water flow is expressed in terms of the sum of these different potentials. The first potential comprises the action of the porous skeleton and the capillary effects. When the scale $\alpha$ is very small and the water is mostly contained in layers or intimately mixed with the micro-skeleton, pressure measurement becomes problematic or impossible [Baker \& Frydman, 2009]. Therefore, in this case, variation of Darcy's coefficient in relation to moisture content cannot be analysed experimentally. For soils, one can try to extrapolate values of Darcy's coefficient from high moisture content values [Van Genuchten, 1980] to low moisture content values. However, simulations of very low moisture content profiles using this procedure are imprecise [Ouedraogo, 2008].

61

Studies on diverse natural media such as rubber [Auria et al 1990], agar gel [Mrani et al. 1995], pasta [Ponsart et al. 2003], and coffee [Ramirez-Martinez et al. 2013] show that a law similar to Fick's law correctly describes the global transfer of water at the $\beta$ scale. The driving force used in these cases, was average moisture content. When moisture content is the only variable parameter, it is always possible, in a simulation, to write the flux of water in terms of moisture content gradient. In the case of Fick's law, an energetic variable such as pressure or chemical potential is replaced by a composition variable: water content. This is possible because of the relationship between energetic variables (pressure or chemical potential) and a composition variable (water content). This relationship is included in the mass transfer coefficient. In a case of composite material, water content is not a good parameter for the description of water transfer [Guillard V. et al. 2003]. A deeper discussion on this subject will be presented later in this article.

Considering that chemical potential is representative of the energetic bond between water and the rest of the media, its use as the driving force for mass transfer in porous media should be preferred [Job et al. 2006]. Moreover, it can replace pressure in situations where pressure measurements are no longer possible. It is also useful in situations where there is a mixture of different phases or electrolytes. At constant temperature, both at the $\alpha$ and $\beta$ scale, the transfer of a chemical species takes place from regions where its chemical potential is higher toward regions where it is lower [Callen, 1985]. At equilibrium, the chemical potential value of a species is uniform throughout the medium at both scales. This equilibrium property allows the measurement of chemical potential for great variety of media (soils, gels, food products), at any moisture content value [Bénet et al., 2012]. Chemical potential measurement can be performed by well-known methods: the tensiometer for the lower absolute values, and the saturated saline solutions method for the higher absolute values. A mechanical method able to measure a wide range of chemical potential values has recently been developed [Ouoba et al., 2010].

The choice of chemical potential to describe mass transfer leads to adopting a thermodynamic approach. Therefore, the phenomenological relationship describing water transfer in structures at the $\alpha$ scale will be set using dissipation. At constant temperature, dissipation is defined as the product of the temperature by the entropy production per unit of volume and time. This relationship will then be extended to study complex media at the $\beta$ scale, particularly, to interpret experimental water transfer results from wood (amaranth) in the radial direction of anisotropy. 


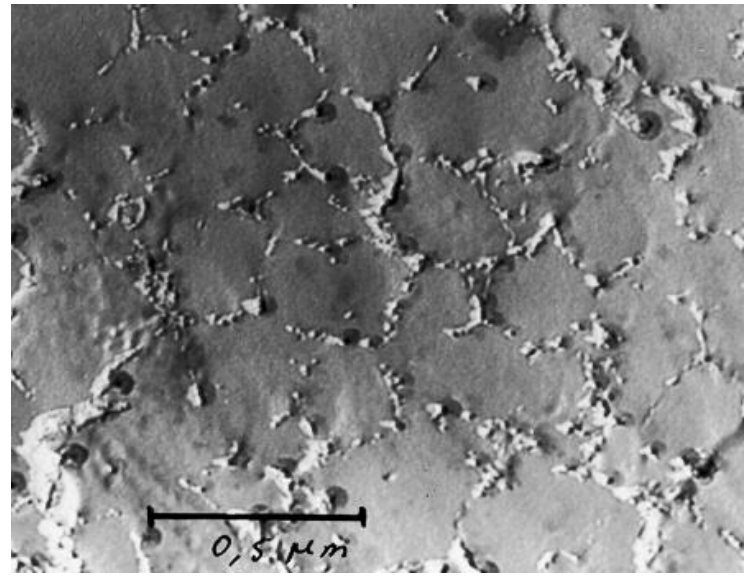

a)

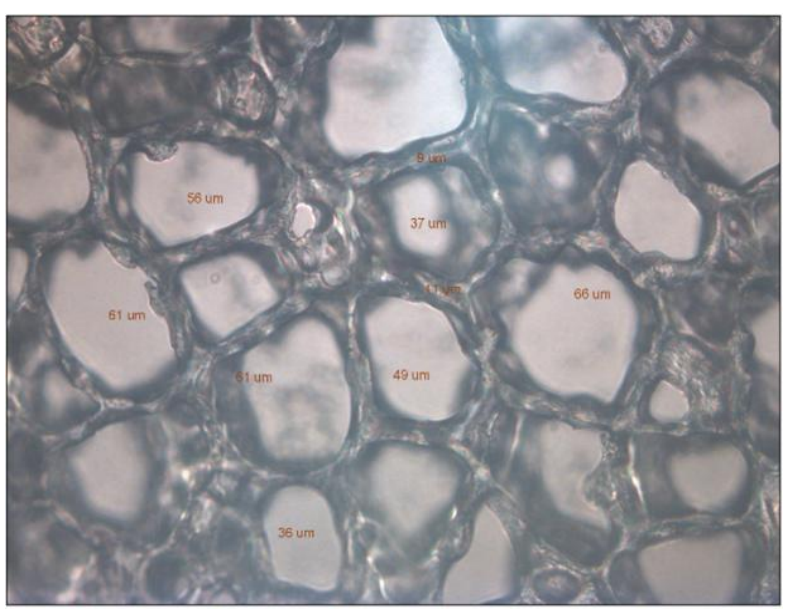

b)

Fig.1: Examples of microstructures of complex media. a) Agar gel b) Coffee bean endosperm.

\section{Thermodynamic equilibrium at $\alpha$ and $\beta$ scales.}

\subsection{Chemical potential of water in complex media.}

The mass chemical potential is defined as the partial derivative of the internal energy $(U)$ of the whole media in relation to the mass of the considered species $\left(m_{i}\right)$, where entropy $(S)$, volume $(V)$ and the concentration of the other constituents $\left(m_{j}\right)$ are kept constant [Guggenheim, 1965]:

$$
\mu_{i}=\left(\frac{\partial U}{\partial m_{i}}\right)_{V, S, m_{j}} ; j \neq i
$$

This thermodynamics function presents the same level of generality as temperature and pressure for pure phases [Job, 2006]. Therefore, chemical potential can also be interpreted from a mechanical point of view in the case of complex media. According to definition (1), the mass chemical potential of a chemical species $i$, for example water, corresponds to the mechanical energy required for the extraction of a unit mass of $i$ in a reversible, adiabatic, constant volume transformation, the other species $j \neq i$ being kept in the media. The $\alpha$ structures can be separated by physical membranes, superficial layers, or simply by free surfaces, like for example, the surface between a liquid and a gas phase. The fundamental property of the chemical potential stipulates that in a natural transformation at constant temperature, a chemical species moves from the regions where its chemical potential is higher toward the regions where it is lower. Movement can occur inside a phase or between phases.

At thermodynamic equilibrium, if a species $i$ is present in several phases $\alpha$ and $\beta$, separated by a permeable membrane only to constituent $i$, we have [Callen, 1985]:

$$
\left(\mu_{i}\right)_{\text {at phase } \alpha}=\left(\mu_{i}\right)_{\text {at phase } \beta}
$$

\subsection{Consequence of the assumption of a local thermodynamic equilibrium.}


131 We are interested in those media where the characteristic dimensions of structures at $\alpha$ are 132 very small compared to the dimensions of the macroscopic objects (grain, cylinders or plates). We will consider these media at a uniform constant temperature. In coffee for example, the ratio between cell size and the grain is of the order of $10^{-3}$. This ratio is of the order of $10^{-4}$ if compared to the cell wall. Considering the difference of ratio values, it is reasonable to suppose that the time to reach water chemical potential equilibrium between two structures at $\alpha$, for example between the cell and the cell wall in the case of a coffee grain, is very fast compared to the characteristic time at which transfer occurs on a macroscopic scale. For example, at the macroscopic scale, the time it takes for water to transfer from the center to the surface of the coffee grain may be of the order of one day [Ramirez, 2011] in industrial drying.

By adopting the hypothesis of local equilibrium, Equation (2) implies that the chemical potential of water $\left(\mu_{w}\right)$ is the same in all the structures contained in contained in a Representative Elementary Volume (REV) at all time. The moisture content of each structure $\left(x_{\alpha}\right)$ varies by mass transfer with the other structures in order to satisfy chemical potential equality. The chemical potential of water in each microstructure is characterized by a relationship between the water activity in the micro structure $\alpha$ and its moisture content $x_{\alpha}$. By adopting free pure water as a reference at the same temperature, this relationship can be written as [Guggenheim, 1965]:

$$
\mu_{w}=\frac{R T}{M_{w}} \ln \left(a_{w \alpha}\left(x_{\alpha}\right)\right), \forall \alpha
$$

153

154

155

156

157

158

160

161

162

163

164

165

166

167 168

$a_{w \alpha}\left(x_{\alpha}\right)$ represents the activity of water vapor in equilibrium with the water of the structure $\alpha$. The relationship between $a_{w \alpha}$ and $x_{\alpha}$ is given by the desorption isotherm of the structure $\alpha$ if the determination of the latter is feasible. The average moisture content of the medium is defined by:

$$
X=\frac{m_{w}}{m_{s}}=\frac{\rho_{w}}{\rho_{s}}=\sum_{\alpha} \frac{x_{\alpha} m_{s \alpha}}{m_{s}}
$$

Where $m_{w}$ is the total mass of water in the REV, $m_{s}$ is the total mass of solid in the REV and $m_{s \alpha}$ the mass of solid in the structure $\alpha$ of the REV with $m_{s}=\sum_{\alpha} m_{s \alpha} \cdot \rho_{w}$ and $\rho_{s}$ are the apparent bulk densities of water and solid phase, respectively, at the $\beta$ scale. In absence of dissolution or chemical reactions, the ratio $m_{s \alpha} / m_{s}$ remains constant, and knowing of the moisture content of the different structures at $\alpha\left(x_{\alpha}\right)$, allows one to calculate the value of $X$ by equation (4) and $\mu_{w}$ by equation (3). These relationships are monotonous, so there is a relationship between $X$ and the water activity which is noted by $a_{w}(X)$ :

$$
\mu_{w}=\frac{R T}{M_{w}} \ln \left(a_{w}(X)\right)
$$


This relationship shows that regardless of the complexity of the structure, if local equilibrium is assumed, the chemical potential of water is a function of the average moisture content, the latter being accessible experimentally.

\subsection{Sorption isotherm}

It is therefore possible to characterize the thermodynamic state of the water at the $\beta$ scale by a single isotherm, using the average moisture content $(X)$. For example, the sorption isotherm for three media is given in Figure 2: wood [Anoua, 1986], agar gel [Mrani, 1995] and coffee [Ramirez-Martinez, 2011].

The GAB model (named after their three authors Guggenheim, Anderson, Boer) describes adsorption on a homogeneous surface, the adsorbed liquid being deposited in successive layers on the surface of the solid. It gives the relationship between the water content of the medium $(X)$ and water activity $\left(\mathrm{a}_{\mathrm{w}}\right)$ [E. Timmermann O. et al, 2001; Escalona et al, 2008].

$$
X=\frac{X_{m} C_{g} K_{g}}{\left(1-K_{g} a_{w}\right)\left(1+\left(C_{g}-1\right) K_{g} a_{w}\right)} a_{w}
$$

The model shows two constants: $\mathrm{Cg}$ associated with the adsorption energy of the first and second layers and $\mathrm{Kg}$ associated with the binding energies of the following layers. $\mathrm{Xm}$ is the water content corresponding to the first adsorbed layer.

Model parameters are calibrated to the experimental isotherms. The values of GAB parameters for wood, agar gel and coffee endosperm are Given in Table 1.

Table 1: Parameters of the GAB model for the wood, agar gel and coffee endosperm.

\begin{tabular}{|l|c|c|c|c|}
\hline & $X_{m}$ & $C_{g}$ & $K_{g}$ & $\partial X / \partial a_{w}(X=0)$ \\
\hline Wood (amaranth) & 0.0626 & 4.71 & 0.851 & 0.251 \\
\hline Agar gel & 12.33 & 17.225 & 0.810 & 1.716 \\
\hline Coffee endosperm & 0.0632 & 12.777 & 0.871 & 0.703 \\
\hline
\end{tabular}

The GAB model gives a good fit for water activities less than 0.9. The final column of Table 1 shows the derivative at the origin of $X$ with respect to $a_{w}$, calculated from (6):

$$
\left(\frac{\partial X}{\partial a_{w}}\right)_{a_{w} \rightarrow 0}=1 /\left(\frac{\partial a_{w}}{\partial X}\right)_{X \rightarrow 0}=X_{m} C_{g} K_{g}
$$

As the water activity of the saturated saline solutions employed are known [Norm NF X 15119,1999 ], it is possible to use equation (5) to establish the relationship between the chemical potential of the water in the medium and the moisture content at equilibrium (Fig 3). 


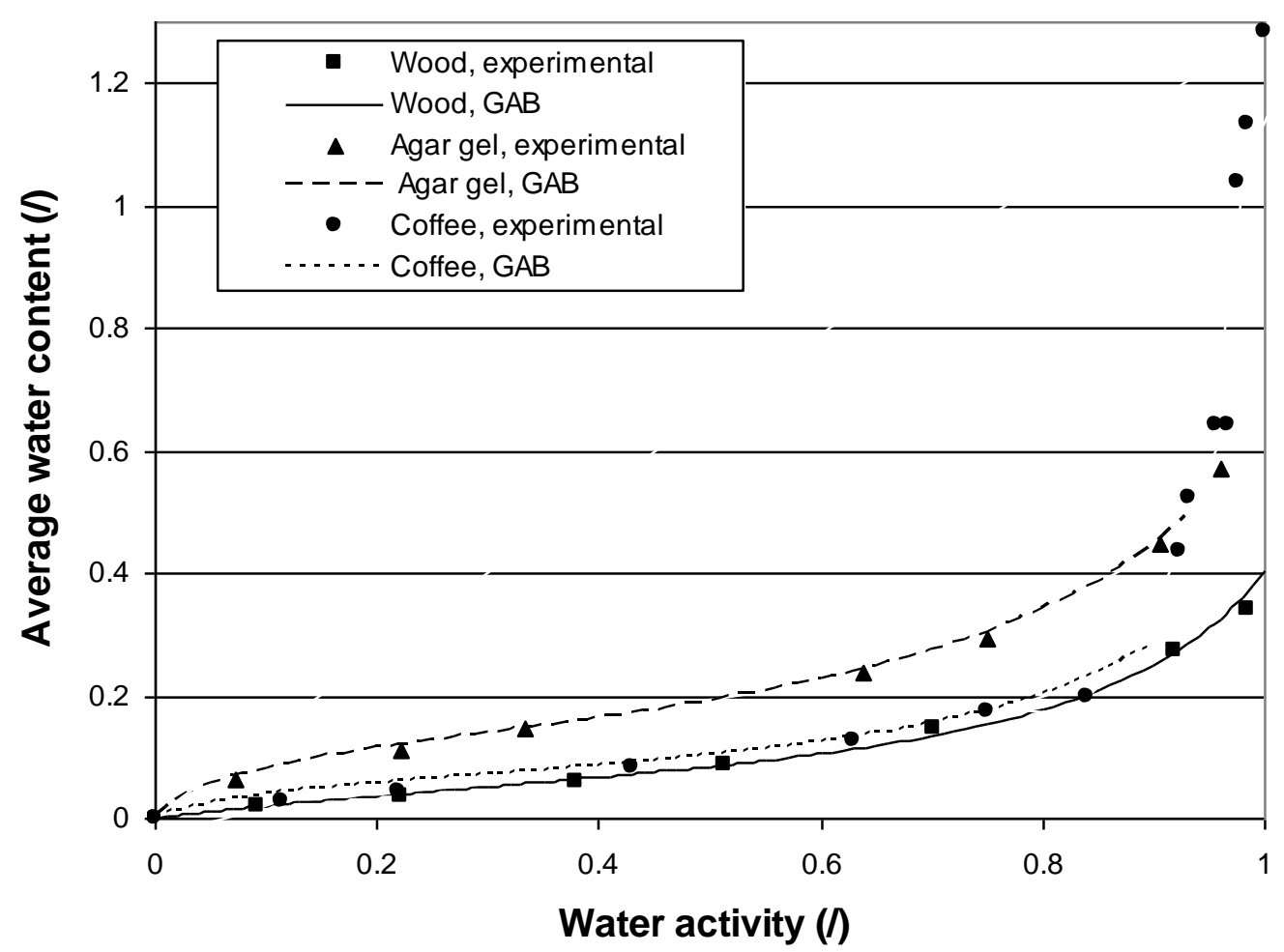

Figure 2. Isothermal desorption of three media: wood (solid squares), agar gel (solid triangles) and coffee (solid circles). The approximations obtained by the GAB model are also represented. For the agar gel, moisture content must be multiplied by 100 .

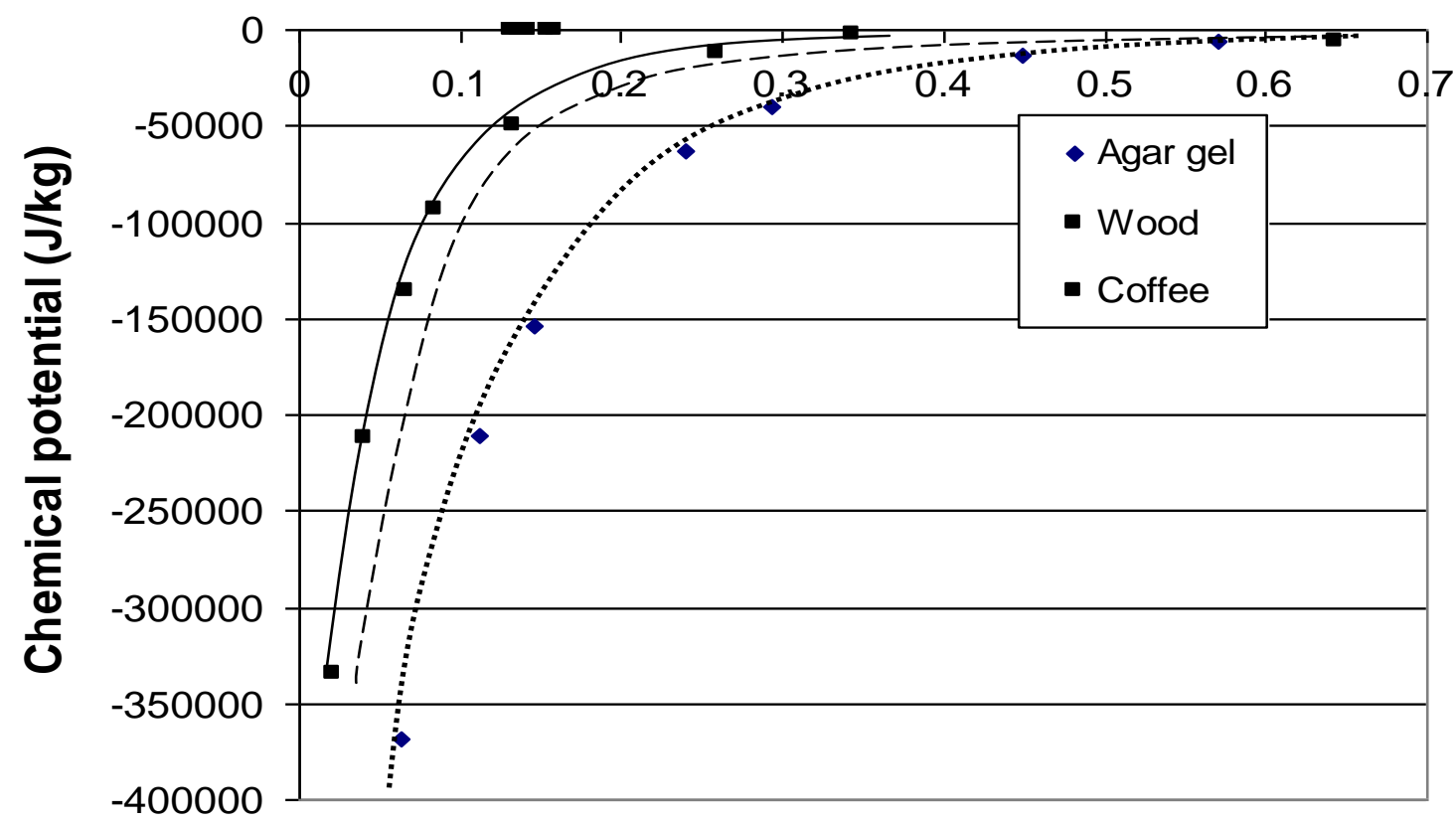

Average water content (/)

Figure 3. Variation of chemical potential as a function of the average moisture content for wood, agar gel and coffee (reference state: free water at the same temperature as the medium). 
According to the definition of chemical potential (1) the partial derivative of the internal energy must be evaluated at constant entropy. This is equivalent to a reversible adiabatic transformation. These conditions are approached during the establishment of an isotherm. The chemical potential can then be matched to the mechanical energy needed to extract the mass unit of the medium.

Like any thermodynamic quantity, the chemical potential is defined using a reference state; in equation (5) it is free pure water at atmospheric pressure and room temperature, the activity is then set to 1 . Under these conditions, according to (5), the chemical potential is negative and tends toward minus infinity when the water content tends to zero. Figure 3 shows that the energy required to extract the unit weight of water and bring it to the reference state increases as the water content decreases.

For pure water which is assumed incompressible, when pressure measurement is possible, relative pressure is connected to chemical potential by: $p_{w}=\rho_{w} \mu_{w}$ [Guggenheim, 1965]. The pressure measurement is not possible in finely divided media like wood or coffee. For example, for a water content below 0.15 , Figure 3 shows that the chemical potential is less than $-50 \mathrm{~kJ} / \mathrm{kg}$. The pressure calculated by the equation above is $500 \times 10^{5} \mathrm{~Pa}$. Theses values are far from the measurement capabilities of the pressure [Baker \& Frydman, 2009]. This justifies the use of the chemical potential to describe water transfer, when water is strongly linked to the solid phase.

\section{Mass transfer at scales $\alpha$ and $\beta$}

In complex media, each structure at $\alpha$ scale is composed of components noted as $\alpha i$. It is assumed that the temperature is uniform and constant, there are no chemical reactions in the media and that the mechanical behavior of the medium is reversible (elastic). Neglecting gravity, dissipation $D_{i r}$ (associated with transport phenomena in all structures), can be expressed as [Prigogine et al.,1951; Bénet et al.,1983; Müller,2001]:

$$
D_{i r}=-\sum_{\alpha} \sum_{i} \rho_{\alpha i} v_{\alpha i}^{k} \mu_{\alpha i, k} \geq 0
$$

Where $\rho_{\alpha i}$ is the bulk density of the component $\alpha i$ and $v_{\alpha i}^{k}$ is the velocity; $\rho_{\alpha i} v_{\alpha i}^{k}$ is the mass flux of the component $\alpha_{i}$. It is assumed that water is the only moving component. The index $w$ is reserved for the water component, therefore dissipation (equation 8) becomes:

$$
D_{w}=-\sum_{\alpha} \rho_{\alpha w} v_{\alpha w}^{k} \mu_{\alpha w, k} \geq 0
$$

According to the hypothesis of local equilibrium, the chemical potential gradient of water is the same for all structures. Dissipation (9) can be written:

$$
D_{w}=-\left(\sum_{\alpha} \rho_{w} v_{w}^{k}\right) \mu_{w, k} \geq 0
$$

Water movement occurs in structures occupying domains of different geometries. It can be assumed that the transport mechanisms in a structure at $\alpha$ scale are independent of phenomena that develop in other structures at $\alpha$ [Prigogine et al., 1951; Bénet et al.; 1983]. 
In consequence, the dissipations $D_{w \alpha}$ due to the movement of water in the different structures $\alpha$ are separately positive:

$$
D_{w \alpha}=-\rho_{\alpha w} v_{\alpha w}^{k} \mu_{w, k} \geq 0
$$

The conditions imposed on this dissipation (positivity away from the equilibrium and equilibrium nullity), are achieved if the mass flux of water into the structure $\alpha$ satisfies the assumption of linearity in the vicinity of the equilibrium [De Groot et al., 1969; Kuiken, 1994].

$$
\rho_{\alpha w} v_{\alpha w}^{k}=-K_{\alpha}\left(x_{\alpha}\right) \mu_{w, k}
$$

The quantity $K_{\alpha}\left(x_{\alpha}\right)$ represents the coefficient of water transfer in the structure $\alpha$. This quantity is positive and depends on the moisture content of the structure $\alpha$. The question is whether we can express $K_{\alpha}\left(x_{\alpha}\right)$ in terms of the average moisture content $X$. From equation (5), knowledge of $X$ provides access to $\mu_{w}$. This value introduced in (3) shows that the moisture content, $x_{\alpha}$, is fixed in all structures $\alpha . X$ being fixed, $x_{\alpha}$ is also defined:

$$
x_{\alpha}=x_{\alpha}(X), \forall \alpha
$$

Equation (13) implies that all the coefficients $K_{\alpha}\left(x_{\alpha}\right)$ of equation (12) can be expressed in terms of $X$. The overall flux of water is expressed by a relation of the form:

$$
\rho_{w} v_{w}^{k}=-\sum_{\alpha} \rho_{w \alpha} v_{w \alpha}=-K(X) \mu_{w, k}
$$

With:

$$
K(X)=\sum_{\alpha} K_{\alpha}\left(x_{\alpha}(X)\right)
$$

Using (5), (14) can be written in terms of average water content gradient:

$$
\rho_{w} v_{w}^{k}=-\rho_{s} D(X) X_{, k}
$$

Setting:

$$
D(X)=K(X) \frac{R T}{\rho_{s} M_{w} a_{w}(X)} \frac{\partial a_{w}}{\partial X}
$$

$$
\frac{\partial \rho_{w}}{\partial t}=\frac{\partial \rho_{s} X}{\partial t}=-\operatorname{div}\left(\rho_{w} v_{w}^{k}\right)
$$


Using equation (16) and considering a uniform and constant bulk density of the solid phase $\rho_{s}$, equation (18) takes the form of a diffusion equation:

$$
\frac{\partial X}{\partial t}=\operatorname{div}\left(D(X) X_{, k}\right)
$$

The coefficient $D(X)$ is expressed in $\mathrm{m}^{2} / \mathrm{s}$. In the above equations, the energetic driving force responsible for the transport (chemical potential gradient) is replaced by another, non energetic driving force related to composition (moisture content gradient). Such substitution is only possible if there is a local equilibrium traduced by a single isotherm linking the chemical potential with the average moisture content as expressed in (5). Also, the bulk density of the solid phase must be uniform and constant.

The variation of the function $\mu_{w}(x)$ is monotone (Fig.5), so an inverse function $X\left(\mu_{w}\right)$ can be defined. Using (14), the mass balance equation (18) can be written as:

$$
\rho_{s} \frac{\partial X}{\partial \mu_{w}} \frac{\partial \mu_{w}}{\partial t}=\operatorname{div}\left(K\left(X\left(\mu_{w}\right)\right) \mu_{w, k}\right)
$$

Equation (20) is, in this form, analogous to the heat equation. It highlights the strong analogy of the temperature $\mathrm{T}$ and chemical potential $\mu_{w}$. This is due to the fact that temperature and chemical potential are defined as the partial derivatives of a single thermodynamic potential, here the internal energy (1). The term $\partial X / \partial \mu_{w}$ is similar to the specific heat and $K\left(X\left(\mu_{w}\right)\right)$ to the thermal conductivity.

\section{Use of the chemical potential of water to describe water transfer in the radial direction of wood on the $\beta$ scale.}

We propose to validate relationship (14) in order to judge the validity of the two assumptions on which it is based: the hypothesis of local equilibrium of all forms of water and the assumption of linearity. Moreover, this study will establish the variation of the transport coefficient $\mathrm{K}(\mathrm{X})$ as a function of the moisture content.

\subsection{Experimental study.}

As the solid skeleton of wood is anisotropic, we limit ourselves to the study of transfer in the radial direction. The wood used in this study is amaranth whose desorption isotherm is shown in Figure 2. The variation of the chemical potential of water as a function of moisture content is given in Figure 3. As the contraction of wood (amaranth) is less than $4 \%$ and the range of the studied moisture content values is less than $20 \%$, the effect of contraction on water transfer is neglected. The opposite is certainly not true.

The experimental setup is shown in Figure 4. Wood samples of 50x25x10 mm were cut from a wood ridge. The dimension 50 corresponds to the radial direction, that of 25 to the transverse direction and that of 10 to the longitudinal direction. Ten samples were placed in a closed chamber in which the water activity was adjusted to 0.20 and the temperature to $60^{\circ}$ C. Only the lower side of the samples was exposed. The other sides were sealed by an 
aluminium film to assure the airtightness of the samples. To assure that water transfer was effectively occurring in the radial direction ( $\mathrm{Ox}$ at Figure 4), a sample was suspended from a balance and its weight was measured continuously. To determine the profiles of moisture content at a given time $\left(t_{i}\right)$, a sample was taken and cut into slices of $1 \mathrm{~mm}$ thickness perpendicularly to the radial direction. After measuring the moisture content of the slices, it was possible to trace the moisture content profiles at different times as shown schematically in Figure 4.

Temperature controlled device

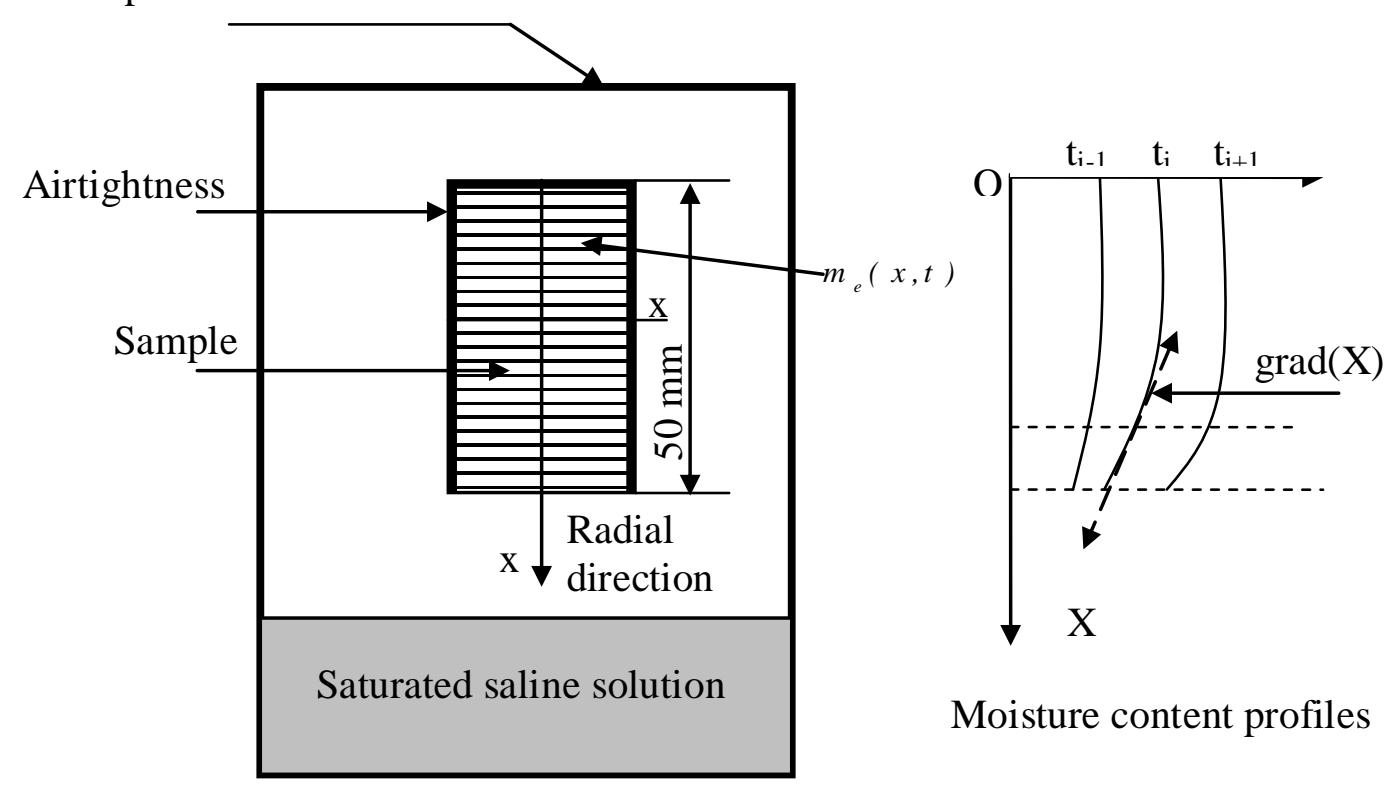

Figure 4. Schematic representation of the experimental device for measuring water flux and moisture content gradient.

The radial flux, at the abscissa $x$, at time $t$ is calculated by:

$$
\rho_{w} v_{w}(t, x) S_{x}=\frac{d}{d t} m_{w}(t, x)=\frac{d}{d t} \int_{0}^{x} X(t, \varsigma) \rho_{s} d \xi
$$

Where $S_{x}$ is the surface perpendicular to $x$ axis, $m_{w}(t, x)$ is the mass of water contained in the sample between the upper surface $(\mathrm{x}=0)$ and the abscissa $x$ at the instant $t, \rho_{s}$ is the bulk density of the wood and $\xi$ an abscissa between 0 and $x$. Relationship (21) gives, at different times, the flux $\rho_{w} v_{w}$ at $x$ from experimental profiles of moisture content. The moisture content gradient is deduced directly from the tangent to the curve $X(t, x)$ (Figure 4). At any given time, the profile of $X$ gives access to the chemical potential profile using the desorption isotherm equation (6) and the equation (5).

For a given abscissa (values: $x=4,6,10,16,20 \mathrm{~mm}$, are adopted) and a given time, water flux $\left(\rho_{w} v_{w}(t)\right)$, the chemical potential gradient $\mu_{w, k}(t)$ and the moisture content $X(t)$ are known. The value of $K$ is calculated for a given moisture content $X$ using (14). The open squares at Figure 6 are all the $\mathrm{K}$ values determined for different abscissas and different times at $60^{\circ} \mathrm{C}$. 
397 Fixing moisture content $X_{0}$, the curve $X(t)$ allows to determine the instant when a moisture content is reached for a fixed abscissa $x$, also values from $\rho_{w} v_{w}\left(X_{0}\right)$ and $\mu_{w, k}\left(X_{0}\right)$ can be deduced. The relationship between water flux and the chemical potential gradient is shown in Figure 5 for $x_{0}=6 \%, 8 \%, 10 \% 12 \%$ and $14 \%$. This figure shows that the obtained relationships, parameterized by $X_{0}$, do not satisfy the linearity assumption perfectly. However, its behaviour is not far from linearity, and is nearly attained in the vicinity of the origin. There is also better linearity at high moisture contents. The curves in Figure 5 are well approximated by quadratic functions. When the water content decreases (eg $\mathrm{X}=0.06$ in Figure 5) the binding energy increases; this hampers the movement of water and the value of $\mathrm{K}$ decreases.

When the chemical potential gradient approaches zero, values of $K\left(X_{0}, \mu_{w, k} \rightarrow 0\right)$ can be deduced from the slopes at the origin. These values at $60^{\circ} \mathrm{C}$ are shown in Figure 6 (solid squares). This figure also shows the values corresponding to the slopes of the curves in Figure 5 for a chemical potential gradient of $10^{7}: K\left(X_{0}, \mu_{w, k}=10^{7}\right)$ (solid triangles).

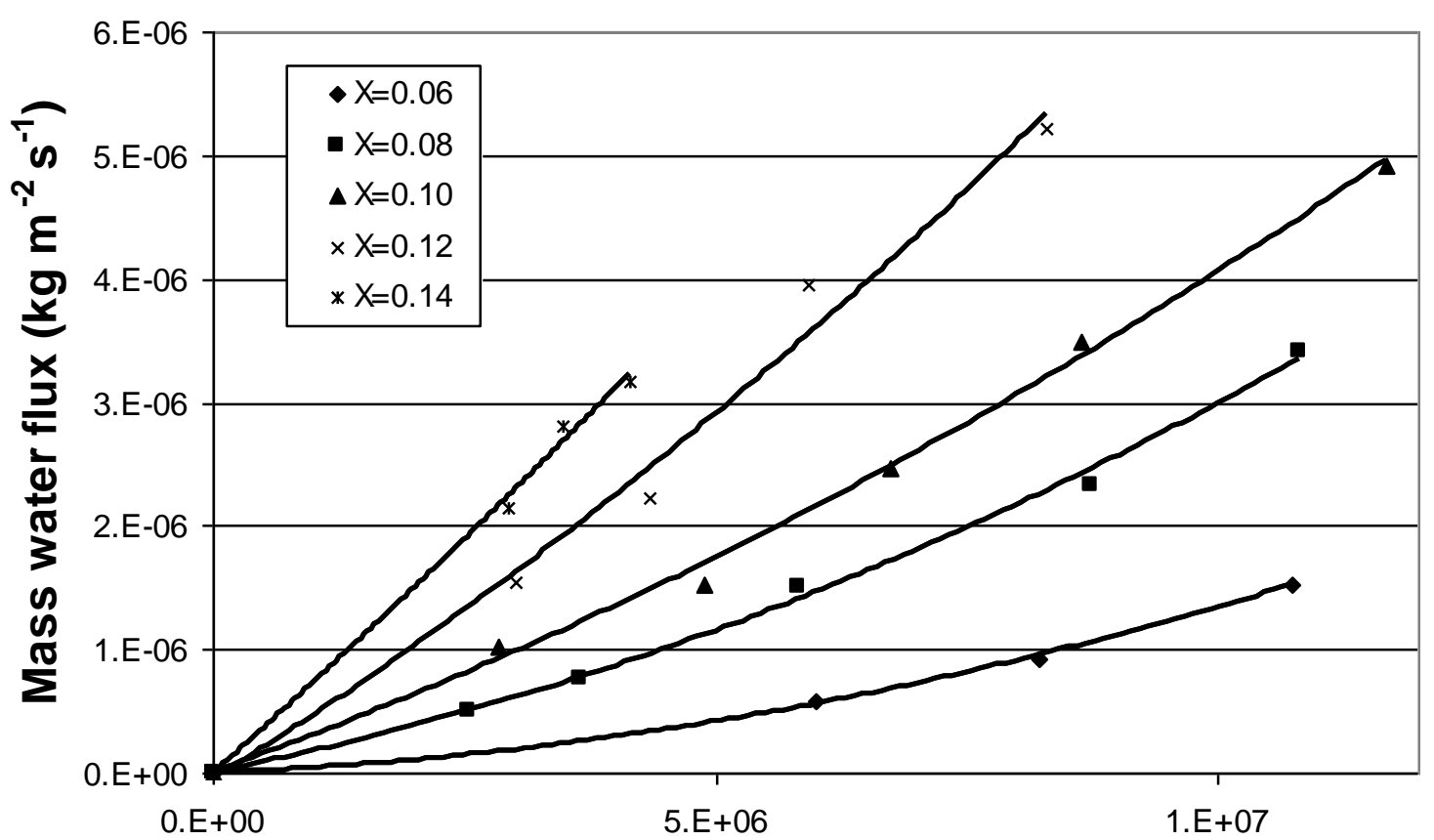

\section{Chemical potential gradient $\left(\mathrm{J} \mathrm{kg}^{-1} \mathrm{~m}^{-1}\right)$}




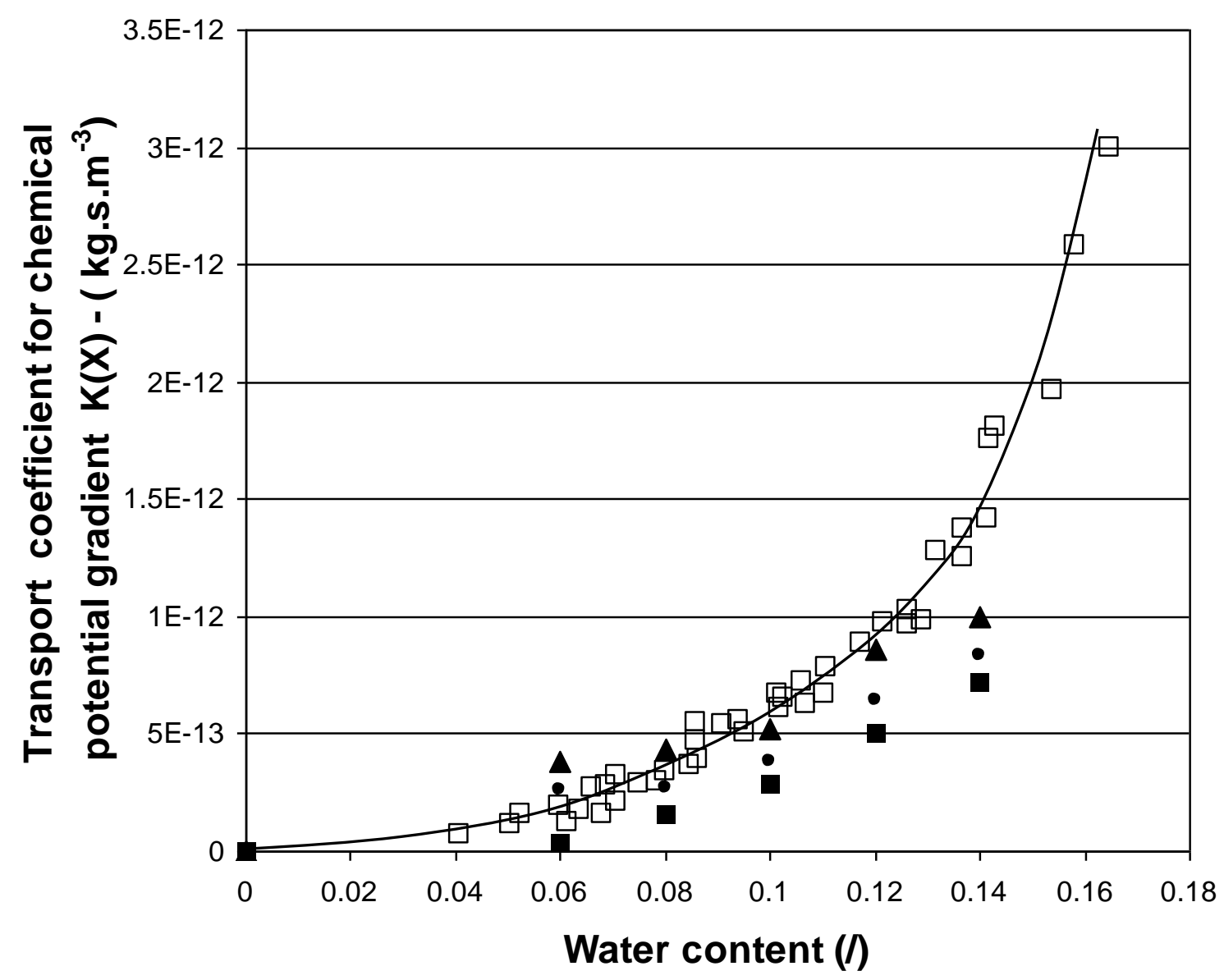

Figure 6. Variation of transport coefficient associated with the chemical potential gradient of water in the case of wood.

We can see that the points corresponding to the values of $K\left(X_{0}, \mu_{w, k} \rightarrow 0\right)$ (solid squares) are located below the points corresponding to measurements at different abscissas $K\left(X, 0 \leq \mu_{w, k} \leq 10^{7}\right)$ (open squares). Points corresponding to the values of $K\left(X_{0}, \mu_{w, k}=10^{7}\right)$ (solid triangles) are similar to the latter. These observations confirm that the linearity assumption is not completely satisfied. However, the curves in Figure 6 are consistent, and related to the curves in Figure 5, they make it possible to model coefficient $K(X)$ in a wide region near the equilibrium. As expected, coefficient $K(X)$ tends to zero as moisture content value decreases. This can be explained by the increasing bonding of water in the wood quantified by an increase of the absolute value of the chemical potential as moisture content decreases.

\section{Conclusion}

When bonding between water and the other phases present in a medium is too strong and pressure measurement becomes impossible, chemical potential can be used to characterize the thermodynamic state of the water. Chemical potential can be measured by different methods even when its absolute value becomes very high. 
The use of a single transport equation to describe water transfer in a complex medium, either by the chemical potential or moisture content gradient, is subjected to the hypothesis of the thermodynamic equilibrium of water in all its forms. In the case of experiments on wood, the consistency of the results shows that the hypothesis of local equilibrium is consistent for the experimental conditions used. The chemical potential can simply translate this assumption, allowing water chemical potential to be defined at the macroscopic scale. Under this condition, the equilibrium average moisture content can be used to define a single desorption isotherm.

Another consequence of the assumption of local equilibrium is that dissipation can be expressed as the product of the sum of the water flux by the gradient of a single chemical potential. It is then possible to describe water transport in the vicinity of the equilibrium as a linear relationship between water flux and the chemical potential gradient. In the case of wood, experiments highlight a moderate nonlinearity. However, experiments were conducted under drastic conditions $\left(\mathrm{T}=60^{\circ} \mathrm{C}\right.$ and $a_{w}=0.2$ ) compared to normal conditions (natural environment or drying process). Even under drastic conditions, there is a small deviation from linearity behavior.

Transport coefficient is nil when the water content is zero. Under this condition, it is easy to extrapolate the experimental results for moisture contents less than 0.04 (Figure 6). The proposed method makes it possible to determine the transport coefficient associated with the chemical potential gradient when liquid-solid interaction is strong. Developed for the case of wood, the method can be extended to other media.

Use of the chemical potential gradient is limited by the two asymptotic behaviours shown in Figure 3. At very low water content, a low water content gradient produces a high chemical potential gradient. The coefficient $\mathrm{K}$ tends to zero and the relation (14) is presented as an indeterminate form, which may introduce instabilities in the calculations. When the water content tends toward saturation, water activity tends to 1 making determination of the isotherm unclear. It is preferable, in this case, to measure the water pressure with a tensiometer and use pressure gradient to describe the transfer of water. The combined use of tensiometer and desorption isotherms allows the chemical potential and water content relationship to be expressed in all the fields of water content (Bénet, JC, et al., 2009)

\begin{abstract}
Auria,R., Bénet, J.-C., Transport de l'eau dans une feuille de caoutchouc naturel pendant la période de séchage à vitesse décroissante, Int. J. Heat Mass transfer, 33 (1990), 1885-1894.
\end{abstract}

Anoua, M., Reconnaissance de coefficients de transfert en milieux poreux: diffusion (bois) et conduction (sol), Thèse Université Montpellier 2, 1986.

Baker, R., Frydman, S., Unsaturated soil mechanics Critical review of physical foundations, Engineering Geology, 106 (2009), 26-39.

Bénet J.C., Jouanna P., Non équilibre thermodynamique dans les milieux poreux non saturés avec changement de phase. Int. J. Heat Mass Transfer, vol. 26, $\mathrm{N}^{\circ} 11$ (1983), 1585-1595 
Bénet, J.-C.,Lozano, A.L., Cherblanc, F., Cousin, B., Phase change of water in a hygroscopic porous medium. Phénomenological relation and experimental analysis for water in soil. $J$.

Bénet, J.-C., Ramirez-Martinez, A., Ouedraogo, F., Cherblanc, F., Measurement of the chemical potential of a liquid in porous media. J.Porous Media, Vol. 15 (11), (2012), 10191029.

Callen, H.,B., 1985, Thermodynamics and an introduction to thermostatistics, John Wiley \& Sons.

Corey, A.T., Klute, A., Application of the concept to soil water equilibrium and transport, Soil. Sci. Soc. Am. J., 49 (1985), 3-11.

De Groot, S.R., Mazur, P., 1969, Non-equilibrium thermodynamics, North-Holland Publishing company, Amsterdam,

Efremov, G., \& Kudra, T., Model-based estimated for time-dependent apparent diffusivity. Drying technology, (2004), 23(12), 2513-2522.

Escalona, I. G., Gommes, C. J., Job, N., Blacher, S., Olivera-Fuentes, C. G., Pirard, J.P., Léonard, A., Water desorption from resorcinol-formaldehyde hydrogels and adsorption in the resulting xerogels, Microporous and mesoporous materials, 117 (2008), 61-66.

Guggenheim, E. A., 1965, Thermodynamique, Dunod, Paris.

Guillard, V., Broyart, B., Bonazzi C., Guilbert, S., Gontard, N., Modelling of moisture transfer in a composite food: dynamic water properties in an intermediate $\mathrm{a}_{\mathrm{w}}$ porous product in contact with $\mathrm{a}_{\mathrm{w}}$ filling, Trans IChemE, Vol. 81, Part A, (2003) 1090-1098.

Job, G., Hermann, F., Chemical potential-a quantity in search of recognition, Eur.J.Phys., 27 (2006), 353-371.

Kuiken, G.D.D., 1994, Thermodynamics of irreversible processes. Wiley \& sons.

Mrani, I., Fras, G., Bénet, J.-C, Microstructure et propriétés hygro-mécaniques du gel d' agar, J.Phys. III France, 5 (1995), 985-998.

Müller, I., Thermodynamics of mixtures and phase field theory, International journal of solids and structures, 38 (2001),1105-1113.

Niato, J.J., Bear,J., Potentials and their role in transport in porous media, Water resources research, 32(2) (1996),225-250.

Norme NF X 15-119, Mesure de l'humidité de l'air - Générateur d'air humide à solutions salines pour l'étalonnage des hygromètres. (1999). 
538 Ouedraogo, F., Cherblanc,F., Naon, B., Bénet, J.-C., Water transfer in soil at low water 539 content. Is the local equilibrium assumption appropriate ? Journal of Hydrology, 492 (2013), $540 \quad 117-127$.

Ouoba, S., Cherblanc, S., Cousin, B., \& Bénet, J.C., A new experimental method to determine the sorption isotherm of a liquid in a porous medium. Enviromental Science \& Technology, 44(15) (2010), 5914-5919.

Ponsart,G., Vasseur, J., Frias, J.M., Duquesnoy, A., Méot, J.M., Modelling of stress due to the shrinkage during drying of spaghetti, Journal of Food Engineering , 57 (2003), 277-285.

Prigogine, I., Mazur, P., Sur deux formulations de l'hydrodynamique et le problème de l'hélium liquide II, Physica XVII, 7 (1951), 661-693.

Ramirez-Martinez, A.,Internal structure and water transport in endosperm and parchment of coffee bean, Thèse Université Montpellier 2, 2011.

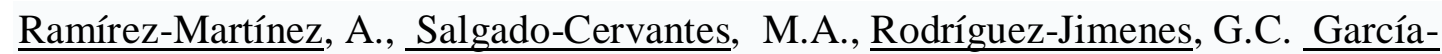

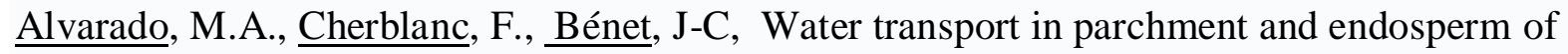
coffee bean, Journal of Food Engineering, 2013 ,Vol.114 (3,) , 375-383.

Timmerman, E.O., Chirife, J., Iglesias, H.A., Water sorption isotherms of foods and foodstuffs: BET or GAB parameters, Journal of Food Engineering, 48 (2001) 19-31. unsatured soils. Annales Geophysicae. 3 (1985), 615-628. 